\title{
Temperature-independent pressure sensor using triangular-shape of Suspended-Core fiber
}

\author{
M. I. Zibaii ${ }^{\mathrm{a}, \mathrm{b}}$, S. Nouri ${ }^{\mathrm{a}}$, J. Sadeghi ${ }^{\mathrm{a}}$, H. Latifi ${ }^{\mathrm{a}, 1}$, P. A. S Joł $\mathrm{e}^{\mathrm{b}}$, \\ K. Schuster ${ }^{c}$, Jens Kobelke ${ }^{\mathrm{c}}$ and O. Frazao ${ }^{\mathrm{b}}$ \\ ${ }^{a}$ Laser \& Plasma Research Institute, Shahid Beheshti University, Evin, Tehran, Iran. \\ bINESC Porto, Rua do Campo Alegre, 687. 4169-007 Porto, Portugal. \\ ${ }^{\mathrm{c}}$ Institute of Photonic Technology Jena, Albert-Einstein-Str. 9,007745 Jena, Germany
}

\begin{abstract}
In this work, fiber in-line Mach-Zehnder Interferometer (MZI) based on triangular-shape suspended core fibers (SCFs) is investigated. The sensitivity of the sensing head was determined for pressure and temperature, respectively. The sensitivities are $0.4 \mathrm{pm} / \mathrm{psi}$ and $13 \mathrm{pm} / \mathrm{psi}$ for longitudinal and radial pressure, respectively. The sensing head was also subjected to temperature and presented very low sensitivity.
\end{abstract}

Keyword: Optical fiber sensors, Microstructured fiber, Mach Zehnder interferometer, pressure.

\section{INTRODUCTION}

In-line fiber interferometers have attracted considerable attention because of their compact design and intrinsic in-line characteristics. Recently, several new types of in-line fiber Michelson and Mach-Zehnder interferometers (MZIs) have been reported, which make use of interferences between the fundamental core mode and the cladding modes [1]. They have been successfully applied as refractive index (RI), temperature, $\mathrm{pH}$, and strain sensors. Such interferometers can be made of a cleaved distal end face optical fiber as one interface and an external surface as the other, accomplished by fusing a hollow core fiber between two SMFs for strain sensing [2]; by connecting two single mode fibers to a short section of multimode fiber for temperature and strain measurement [3]; or by using a hybrid structure composed by a photonic crystal fiber spliced to a hollow core fiber which in turn is spliced to a SMF, or even by fusing a small length of Suspended-core fiber (SCF) to the end of a cleaved SMF for strain and temperature measurement [4].The advent of microstructured optical fibers (MOFs) has opened up a new range of novel fiber-based sensors. It is well known that in a solid-core MOF a fraction of the modal field is located within the air-holes of the cladding. This kind of structure enables a considerable enhancement in fiber design flexibility, providing a new breach for new and enhanced sensing solutions. The SCF can be differentiate from other of MOF class by having relatively large holes that surround a small core (few $\mathrm{m}$ of diameter) which looks suspended along the fiber axis by small width silica walls. The SCFs can be made of a single material, in such case silica, and use for strain and temperature sensing based in a Sagnac and Fabry-Perot interferometer [5]. More recently, opening one of the holes of the MOF was exploited to maximize the interaction of light for evanescent field sensing or for bio-chemical application [6].

In this work, the pressure and temperature sensitivity of in line MZI setups based on triangular-SCF was studied. The results show that the SCF has enhanced pressure sensitivity due to a high air-fraction in the cladding.

\section{EXPERIMENTS AND DISCUSSION}

\section{2-1 Sensor Fabrication}

The sensing element consists in a MZI based on SCF spliced between single mode fibers. The essence of this design is to couple the light from the fundamental core mode into the higher-modes and vice versa. Since the core mode and the highermodes have different propagation constants, the optical path difference (OPD) between them leads to interference

${ }^{1}$ E-mail address: latifi@cc.sbu.ac.ir ; phone (+98) 2129904034; fax (+98) 299022431775 
in the transmission spectra. Furthermore, the core mode and the higher-modes exhibit different responses to various environmental factors, such as surrounding RI, temperature, and strain. Thus, the transmission spectra change when the OPD between the core mode and the higher-modes is altered. The fabrication of the MZI was made by splicing a SMF to a short length of SCF, with its end cleaved (inset figure 1(a)). The total loss of the splice was $3 \mathrm{~dB}$. The SCF was fabricated at the IPHT (Institute of Photonics Technology, Jena, Germany) and is formed by three holes with a diameter of each $52 \mu \mathrm{m}$. The core and the cladding have diameters of $4.4 \mu \mathrm{m}$ and $127 \mu \mathrm{m}$, respectively. The core has a slightly triangular shape due to the cladding asymmetry originated during the fabrication process [7]. The MZI has $100 \mathrm{~mm}$ of length and presents the output spectrum presented in figure 1(b), when illuminated by a superluminescent Diode.
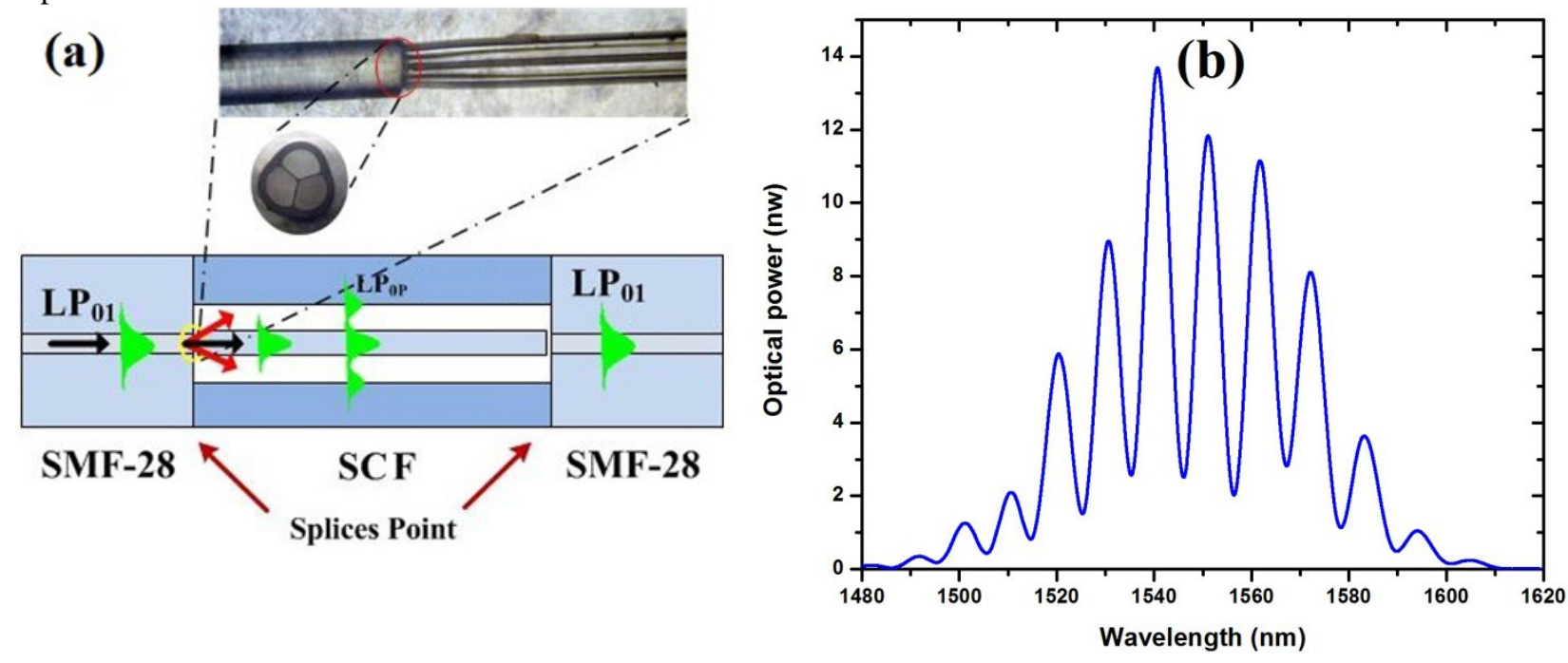

Figure 1. (a) A schematic of the MZI as a sensing head and the cross section microscopic image of SCF, and (b) spectral response of MZI-SCF

\subsection{Experiments}

To determine the longitudinal pressure sensitivity of the MZI-SCF, it was placed inside a high pressure vessel and the SMF-28 was sealed to cap of a high pressure vessel and then hydrostatic pressure was applied. The vessel was pressurized by injection of the water by a high pressure unit. Figure 2(a) shows the experimental setup. To illuminate the system a broadband optical source with a bandwidth of $70 \mathrm{~nm}$ and a central wavelength of $1550 \mathrm{~nm}$ was used. The MZI-SCF output arrived to an optical spectrum analyzer (OSA-86142B) with a maximum resolution of $10 \mathrm{pm}$.
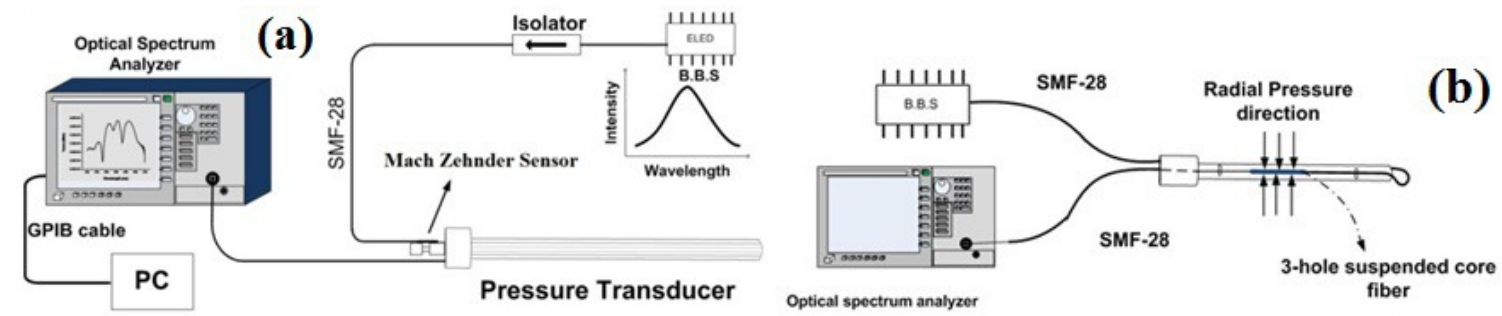

Figure 2. (a) Schematics of experimental set up for longitudinal pressure measurement, and (b) experimental setup for measuring radial pressure measurement.

The longitudinal pressure sensitivity of the bare optical fiber is low and an appropriate design of mechanical transducer has been developed for high pressure applications to convert hydrostatic pressure into longitudinal strain. The complete principle of operation can be found in [8]. In our experiment, the MZI-SCF sensor was bonded to a mechanical transducer. The transducer converts the fluid pressure into fiber strain. Pressure was applied in a range from 0 psi up to 5000 psi (in comparison to atmospheric pressure) and measured using a high precision pressure gauge ( $\pm 5 \mathrm{psi}$ accuracy). The test was done at room temperature. Additionally, this sensing head was also characterized for temperature variation. 

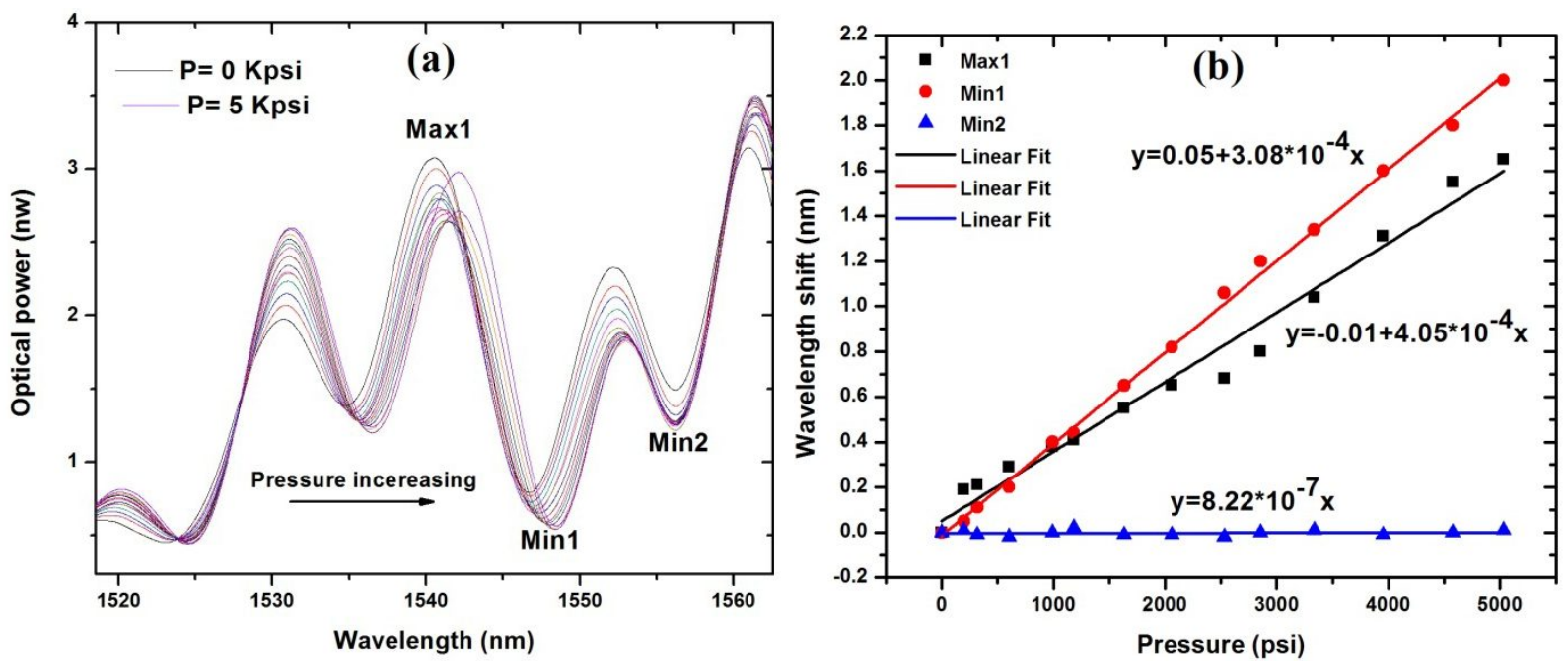

Figure 3. (a) Spectral shift of MZI for 0 to 5 Kpsi (0-345 bar) in longitudinal pressure test, and (b) sensitivity of sensor for

\section{Max1, Min1, and Min2}

The pressure and temperature response of the sensor were obtained by monitoring the measurand induced shift of the MZI-SCF spectrum. In figure 3(a) the results obtained for spectral shift of MZI for 0 to 5 Kpsi (0-345 bar) presented. It was experimentally measured the wavelength shift of transmission signal (Max1, Min1, and Min2) with the pressure (see figure 3). Figure 4 shows the plots of the wavelength shift of transmission Max1, Min1, and Min2 versus the pressure. A linear variation is observed with slopes of $3.08 \times 10^{-4} \mathrm{~nm} / \mathrm{psi}, 4.05 \times 10^{-4} \mathrm{~nm} / \mathrm{psi}$, and $8.22 \times 10^{-7} \mathrm{~nm} / \mathrm{psi}$ for Max1, Min1, and Min2, respectively.
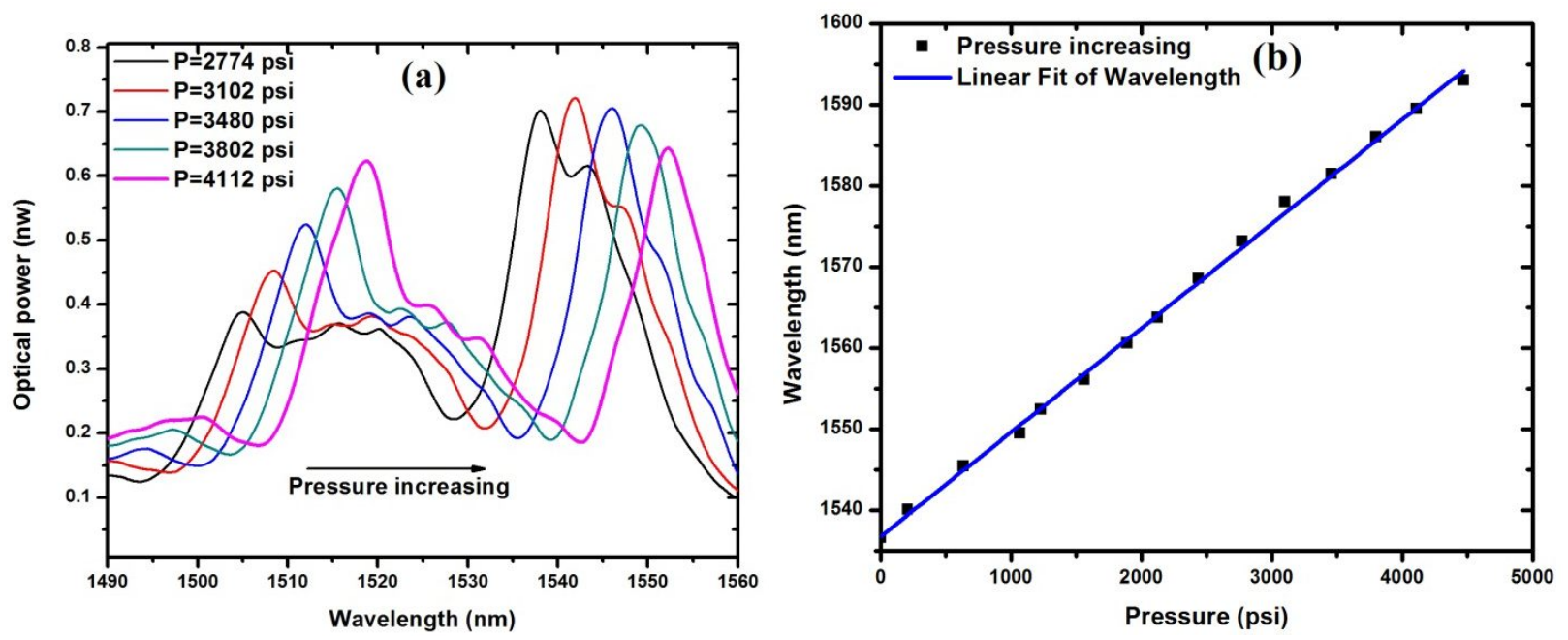

Figure 4. (a) Spectral shift of MZI for 0 to 5 Kpsi (0-345 bar) in radial Pressure test, and (b) sensitivity of sensor for radial pressure measurement for increasing and decreasing of pressure.

To apply radial hydrostatic pressure to the MZI-SCF, the sensor was fixed on the tube surface and placed inside the high pressure vessel. The vessel input/output fibers were sealed as indicated before. Schematic of experimental setup for applying radial pressure is shown in figure 2(b). Radial pressure sensitivity of the MZI-SCF sensors has been measured by increasing the pressure from 0 psi to 5000 psi. Spectral response of sensor for five different pressures is shown in figure 4(a). The pressure sensitivity MZI-SCF sensor has been shown in figure 4(b). The linear fitting of the graph gives pressure sensitivity equal to $0.013 \mathrm{~nm} / \mathrm{psi}$. With a $10-\mathrm{pm}$ resolution (maximum resolution of OSA), the minimum detection of the sensing head at room temperature was for radial pressure calculated to be $0.77 \mathrm{psi}$ for radial pressure measurement. 
The MZI-SCF sensor was also placed in an oven where the temperature was set from room temperature up to $90{ }^{\circ} \mathrm{C}$, with a variation lower than $0.1^{\circ} \mathrm{C}$. Spectral response of sensor towards temperature variations is shown in figure 5(a). The results obtained for wavelength shift versus temperature were exhibited in figure 5(b). In this case, a linear dependence is observed with a slope of $\sim 0.003 \mathrm{~nm} /{ }^{\circ} \mathrm{C}$. The result was compared with fiber Bragg grating (FBG) and it indicated that the MZI-SCF would be practically insensitive to temperature.
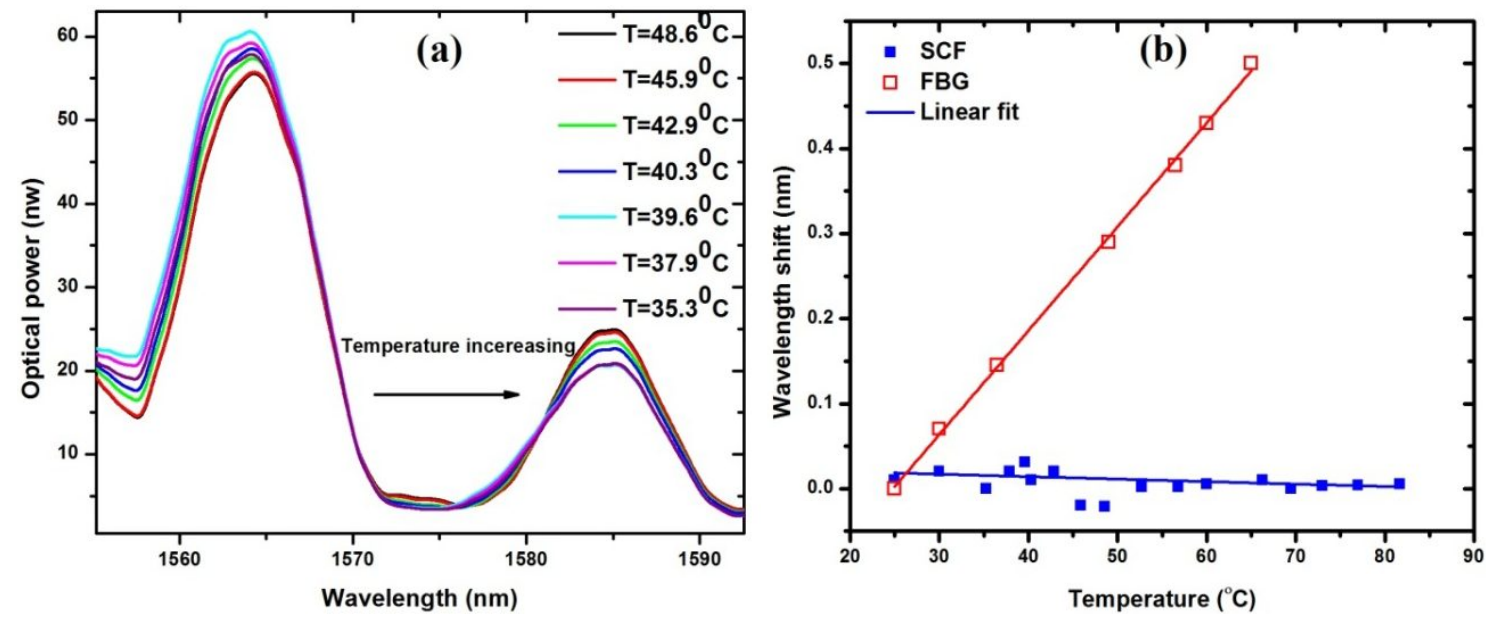

Figure 5. (a) Spectral response of sensor for changing temperature from $35.3^{\circ} \mathrm{C}$ to $48.6{ }^{\circ} \mathrm{C}$, and (b) sensitivity of sensor for temperature measurement comparing with FBG. Sensitivity of MZI based on SCF for $10 \mathrm{~mm}$ sensor length is $0.003 \mathrm{~nm} /{ }^{\circ} \mathrm{C}$.

\section{SUMMERY AND CONCLUSION}

In this work a fiber in line Mach Zehnder interferometer based on pure silica triangular-SCF was proposed and characterized. Longitudinal and radial pressure sensitivities with $0.4 \mathrm{pm} / \mathrm{psi}$ and $13 \mathrm{pm} / \mathrm{psi}$, were respectively achieved. With a 10-pm resolution for the wavelength shift detection our OSA, detection limit of our device at room temperature for radial pressure measurement was calculated to be $0.77 \mathrm{psi}$.

\section{REFERENCES}

[1] Wei, T., Lan, X., and Xiao, H., "Fiber inline core-cladding-mode Mach-Zehnder interferometer fabricated by two-point $\mathrm{CO}_{2}$ laser irradiations,” IEEE Photon. Technol. Lett., 21(100, 669-671 (2009).

[2] De-Wen, D., Yun-Jiang, R., Lai-Cai, x., Tao, Z., Di, W., Jun, Y., “In- fiber fabry-Perot and Mach-Zehnder interferometers based on hollow optical fiber fabricated by arc fusion aplacing with small lateral offsets," Optic. Comm. 248 (22), 5311-5314 (2011).

[3] Villatoro, J., and Monzón-Hernández, D., "Low-cost optical fiber refractive- index sensor based on core diameter mismatch,” J. Lightw. Technol., 24(3), 1409-1413 (2006).

[4] Frazão, O., Silva, S., F., O., Viegas, J., Baptista, J., M., Santos, J., L., Kobelke, J., and Schuster, K., “All fiber Mach-Zehnder interferometer based on suspended twin-core fiber," IEEE Photon. Technol. Lett., 22(17), 1300-1302 (2010).

[5] Aref, S.,H., Zibaii, M.,I., Kheiri, M., Porbeyram, H., Latifi, H., Araújo, F.,M., Ferreira, L.,A., Santos, J.,L., Kobelke, J., Schuster, K., Frazão, O., "Pressure and temperature characterization of two interferometric configurations based on suspended-core fibers,” J. Optics Comm. 285, 269-273 (2012).

[6] Coscelli, E., Sozzi, M., Poli, F., Passaro, D., Cucinotta, A.,Stefano Selleri, Corradini, R., and Marchelli, R., “Toward a highly specific DNA biosensor: PNA-modified suspended-core photonic crystal fibers,” J. Sel. Topics Quantum Electron., 16( 4) , 967-972 (2010).

[7] Pinto, O. Frazão, A., M., R., Santos, J., L., Lopez-Amo, M., Kobelke, J., and Schuster, K., "Interrogation of a suspended-core Fabry-Perot temperature sensor through a dual wavelength raman fiber laser," J. Lightwave Technol. 28(21), 3149-3155 (2010).

[8] Latifi, H., Aref, S., H., Afshari , M., and Zibaii, M.,I., “ Fiber optic fabry perot pressure sensor for downhole applications,” J. Optic Comm .269, 322-330 (2007). 